\title{
Usefulness of TI-scout images in the assessment of late gadolinium enhancement in children
}

\author{
Badr Bannan', Julien Aguet ${ }^{1}$, Aswathy Vaikom House ${ }^{2}$, Navjot Gill1', Vivian P. Tassos' ${ }^{1}$, Afsaneh Amirabadi ${ }^{1}$, \\ Mike Seed ${ }^{1,2}$, Christopher Z. Lam ${ }^{1}$ and Shi-Joon Yoo ${ }^{1,2^{*}}$
}

\begin{abstract}
Background: Cardiovascular magnetic resonance (CMR) late gadolinium enhancement (LGE) requires identification of the normal myocardial nulling time using inversion time (TI)-scout imaging sequence. Although TI-scout images are not primarily used for myocardial assessment, they provide information regarding different signal recovery patterns of normal and abnormal myocardium facilitating identification of LGE in instances where standard LGE images alone are not diagnostic. We aimed to assess the diagnostic performance of TI-scout as compared to that of standard LGE images.
\end{abstract}

Methods: CMR studies with LGE imaging in 519 patients (345 males, 1-17 years) were reviewed to assess the diagnostic performance of LGE imaging in terms of the location of LGE and the pathologic entities. The diagnostic performance of the TI-scout and standard LGE imaging was classified into four categories: (1) equally diagnostic, (2) TI-scout superior to standard LGE, (3) standard LGE superior to TI-scout, and (4) complementary, by the consensus of the two observers.

Results: The study cohort consisted of 440 patients with negative LGE and 79 with evidence for LGE. For a negative diagnosis of LGE, TI-scout and standard LGE images were equally diagnostic in $75 \%$ of the cases and were complementary in $12 \%$. For patients with LGE, TI-scout images were superior to standard LGE images in $52 \%$ of the cases and were complementary in 19\%. The diagnostic performance of TI-scout images was superior to that of standard LGE images in all locations. TI-scout images were superior to standard LGE images in 11 of 12 (92\%) cases with LGE involving the papillary muscles, in $7 / 12$ (58\%) cases with subendocardial LGE, and in 4/7 (57\%) cases with transmural LGE. Tl-scout images were particularly useful assessing the presence and extent of LGE in hypertrophic cardiomyopathy (HCM). Tl-scout was superior to standard LGE in 6/10 (60\%) and was complementary in 3/10 (30\%) of the positive cases with HCM.

Conclusions: TI-scout images enhance the diagnostic performance of LGE imaging in children.

Keywords: Late gadolinium enhancement, Magnetic resonance, Myocardium, Fibrosis, TI-scout, Look-Locker

\footnotetext{
${ }^{*}$ Correspondence: shi-joon.yoo@sickkids.ca

1 Department of Diagnostic Imaging, The Hospital for Sick Children,

University of Toronto, 555 University Avenue, Toronto, ON M5G1X8, Canada

Full list of author information is available at the end of the article
}

\section{Background}

Cardiovascular magnetic resonance (CMR) late gadolinium enhancement (LGE) in has become a gold standard technique for non-invasive assessment of myocardial infarction and fibrosis [1]. LGE imaging requires precise prescription of the inversion time (TI) when the signal of the normal myocardium is nulled $>10$ min after original author(s) and the source, provide a link to the Creative Commons licence, and indicate if changes were made. The images or other third party material in this article are included in the article's Creative Commons licence, unless indicated otherwise in a credit line to the material. If material is not included in the article's Creative Commons licence and your intended use is not permitted by statutory regulation or exceeds the permitted use, you will need to obtain permission directly from the copyright holder. To view a copy of this licence, visit http://creativecommons.org/licenses/by/4.0/. The Creative Commons Public Domain Dedication waiver (http://creativeco mmons.org/publicdomain/zero/1.0/) applies to the data made available in this article, unless otherwise stated in a credit line to the data. 
the injection of a gadolinium-based contrast agent. The TI nulling time of normal myocardium is typically assessed using a modified Look-Locker TI-scout sequence (MOLLI) [1, 2]. Standard LGE imaging is then performed to produce optimal contrast between the healthy and pathologic myocardium using the identified TI nulling time. Although TI-scout imaging is not primarily used for clinical myocardial assessment, it provides valuable information regarding the kinetics of gadolinium in normal and abnormal myocardium [2, 3]. In our day-to-day practice, we found that TI-scout images are frequently helpful or even played a primary role in the identification of abnormal myocardial regions, especially when LGE is mild or heterogeneous, or involved the subendocardial or subepicardial region.

Based on our clinical experience, we hypothesized that TI-scout imaging enhanced the diagnostic performance of LGE imaging in children. In this retrospective study, we aimed to compare the diagnostic performance of the TI-scout images with that of the standard LGE images in the identification of abnormal myocardium.

\section{Methods}

With the approval by our institutional Research Ethics Board, we identified 598 consecutive patients who underwent CMR with LGE imaging between January 2016 and December 2017. Among them, we excluded 79 patients whose TI-scout and standard LGE images were not optimum for proper assessment of LGE. The reasons for nondiagnostic quality included artefact from body motion or irregular breathing pattern (79\%), poor patient's cooperation related to medication such as adenosine and atropine (21\%), metallic artefact (6\%) and others. We retrospectively assessed the images and medical records of the remaining 519 cases (345 males, 1-17 years). All CMR was performed on a 1.5 CMR system (Avanto, Siemens Healthineers, Erlangen, Germany) using the parameters shown in Table 1. LGE was performed approximately $10 \mathrm{~min}$ after an intravenous injection of $0.3 \mathrm{mMol} / \mathrm{kg}$ of gadobenate dimeglumine $(\mathrm{n}=83)$ or $0.2 \mathrm{mMol} / \mathrm{kg}$ of gadobutrol $(n=336)$ at (Fig. 1). TI-scout images were acquired in mid-ventricular short-axis plane using a MOLLI sequence. Standard LGE images were acquired in multiple short-axis and axial planes using segmented FLASH (fast low angle shot magnetic resonance imaging) and single-shot balanced steady state free precession (bSSFP) sequences. Additional TI-scout images were also obtained in multiple short-axis, 4-chamber and 2-chamber planes in patients with suspected LGE at standard LGE images.

The presence, location and extent of LGE were analyzed by comparing the findings seen in the pairs of TI-scout and standard LGE images obtained in the corresponding imaging planes. Postoperative fibrosis such as LGE of the right ventricular (RV) outflow tract in repaired tetralogy of Fallot and equivocal LGE of the RV insertion point to the ventricular septum were not regarded as positive findings. When LGE was identified, its location was classified as subepicardial, mid-myocardial, subendocardial, transmural, papillary muscles and mixed locations. The positive or negative diagnosis of LGE was made by reviewing all available images. The diagnostic performance of TI-scout and LGE imaging was graded as: (1) equally diagnostic, (2) TI-scout superior to standard LGE, (3) standard LGE superior to TI-scout, or (4) complementary for final diagnosis, with the knowledge of the positive or negative diagnosis of LGE for each case. "Complementary" was defined as the situation where the final diagnosis was able to be reached more convincingly by reviewing the images from both sequences than by

Table 1 Technical parameters

\begin{tabular}{|c|c|c|c|}
\hline Parameter & Modified Look-Locker Tl-scout & Standard LGE (segmented PSIR-FLASH) & $\begin{array}{l}\text { Standard LGE } \\
\text { (single-shot PSIR- } \\
\text { bSSFP) }\end{array}$ \\
\hline ECG gating & Throughout R-R interval & Mid-late diastole & Mid-late diastole \\
\hline ECG triggering & Every 2 R-R intervals & Every 2 R-R intervals & Every 2 R-R intervals \\
\hline Breath-hold & No & Yes, if cooperative & No \\
\hline Acceleration factor & 2 & No & 2 \\
\hline Field of view & $250-350 \mathrm{~mm}$ & $250-350 \mathrm{~mm}$ & $250-350 \mathrm{~mm}$ \\
\hline Slice thickness & $7 \mathrm{~mm}$ & $6 \mathrm{~mm}$ & $6 \mathrm{~mm}$ \\
\hline Pixel size & $1.3 \times 1.3 \mathrm{~mm}$ & $1.3 \times 1.3 \mathrm{~mm}$ & $1.8 \times 1.8 \mathrm{~mm}$ \\
\hline Flip angle & $30^{\circ}$ & $45^{\circ}$ & $40^{\circ}$ \\
\hline $\mathrm{TR} / \mathrm{TE}$ & $2.93 / 1.25 \mathrm{~ms}$ & $3.04 / 1.26 \mathrm{~ms}$ & $2.57 / 1.09 \mathrm{~ms}$ \\
\hline Signal averages & 1 & 1 for breath-hold, 3 for uncooperative patients & 1 \\
\hline
\end{tabular}

ECG electrocardiographic, FLASH fast low angle shot, PSIR phase-sensitive inversion recovery, bSSFP balanced steady-state free precession 


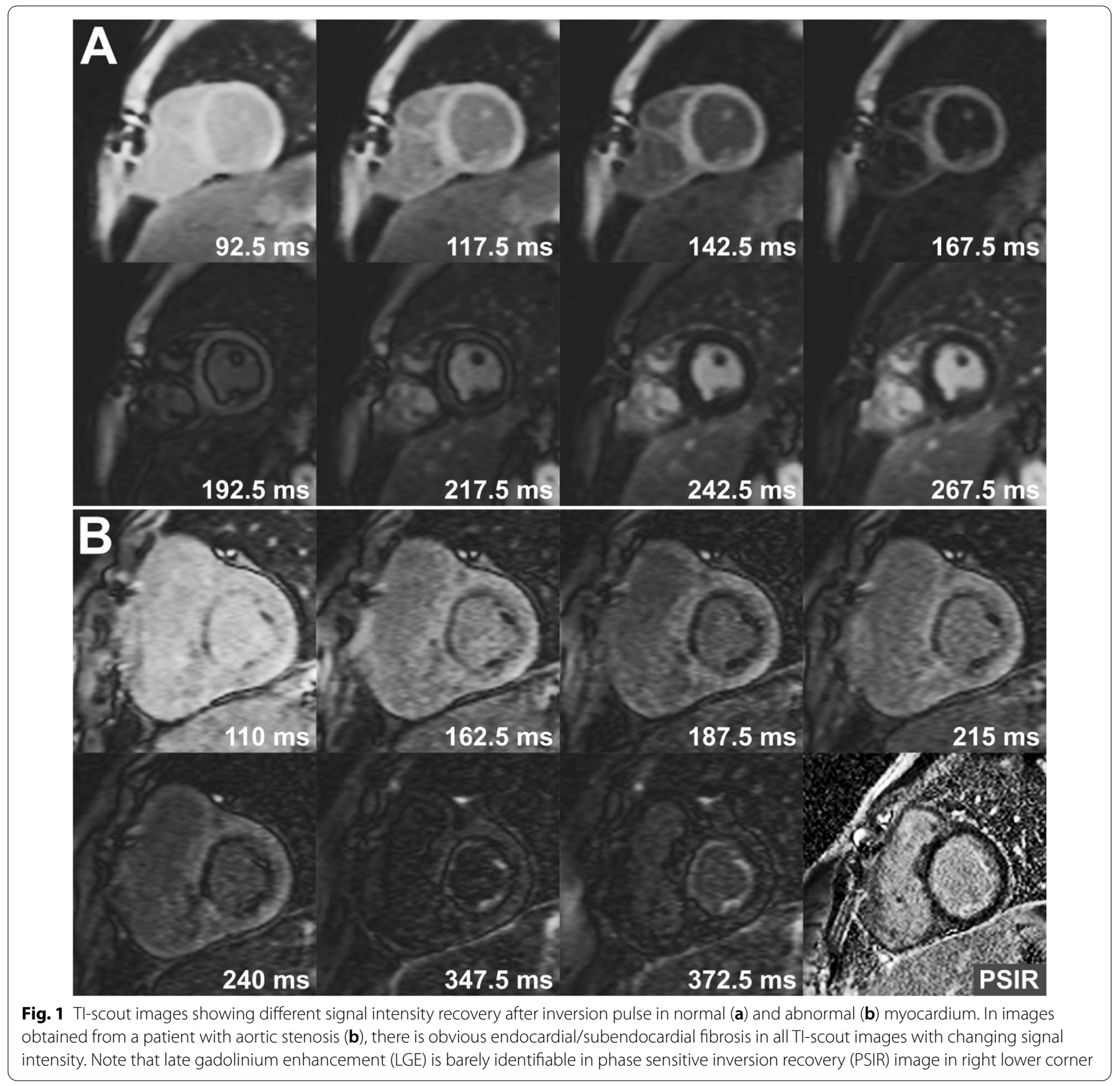

reviewing the images of one sequence alone. The diagnosis of LGE, the location of LGE and the diagnostic performance of the LGE sequences were assessed qualitatively. In those cases where the perception and grading were difficult, the decision was reached after reassessment and consensus between the two observers (BB and SJY).

All statistical analyses were performed using SPSS (version 26.0, Statistical Package for the Social Sciences, International Business Machines, Inc., Armonk, New York, USA). Descriptive analysis was performed for demographic and clinical variables. For quantitative variables, data were expressed as median (range). Dichotomous variables were expressed as $\mathrm{n}$ (\%). Comparison between groups were performed using non-parametric tests (Chi-square). A P-value of less than 0.05 (2-sided) was considered statistically significant.

\section{Results}

The study cohort consisted of 519 patients, 440 with negative LGE and 79 with LGE. The patient characteristics are summarized in Tables 2 and 3. The diagnostic 
Table 2 Patient demographics

\begin{tabular}{ll}
\hline Parameters & Distribution \\
\hline Age, median (range), years & $11(0-17)$ \\
Sex & Male: $345(66.5 \%)$ \\
Weight median (range), kg & $35.5(2.5-139)$ \\
Body surface area, median (range), $\mathrm{m}^{2}$ & $1.19(0.18-2.86)$ \\
General anaesthesia for CMR, $\mathrm{n}(\%)$ & $145(27.9 \%)$
\end{tabular}

Table 3 Disease categories

\begin{tabular}{llll}
\hline Category & LGE negative & LGE positive & Total \\
\hline Unrepaired congenital heart diseases & 44 & 6 & $50(10 \%)$ \\
Repaired congenital heart diseases & 223 & 25 & $248(48 \%)$ \\
Aortic valvular diseases & 19 & 3 & $22(4 \%)$ \\
Anomalous coronary arteries & 8 & 0 & $8(2 \%)$ \\
Hypertrophic cardiomyopathy & 22 & 10 & $32(6 \%)$ \\
Cardiomyopathy or myocarditis (excluding hypertrophic & 86 & 31 & $117(23 \%)$ \\
cardiomyopathy) & 3 & 0 & $3(1 \%)$ \\
Heart transplantation & 6 & 1 & $7(1 \%)$ \\
Cardiac tumors & 29 & 3 & $32(6 \%)$ \\
Systemic vascular diseases & $440(85 \%)$ & $79(15 \%)$ & 519 \\
Total & & & \\
\hline
\end{tabular}

LGE late gadolinium enhancement

Table 4 Diagnostic performance of TI-scout versus standard late gadolinium enhancement in all cases

\begin{tabular}{lrlllr}
\hline & Equally diagnostic & $\begin{array}{l}\text { Tl-scout superior } \\
\text { to standard LGE }\end{array}$ & $\begin{array}{l}\text { Standard LGE superior } \\
\text { to Tl-scout }\end{array}$ & Complementary & Total \\
\hline LGE negative & $330(75 \%)$ & $18(4 \%)$ & $40(9 \%)$ & $52(12 \%)$ & $440(84.8 \%)$ \\
LGE positive & $9(11 \%)$ & $41(52 \%)$ & $14(18 \%)$ & $15(19 \%)$ & $79(15.2 \%)$ \\
Total & $339(65 \%)$ & $59(11 \%)$ & $54(10 \%)$ & $67(13 \%)$ & $519(100 \%)$ \\
\hline
\end{tabular}

LGE late gadolinium enhancement, $T$ I inversion time

Table 5 Diagnostic performance of TI-scout versus standard LGE images in LGE positive cases by disease categories

\begin{tabular}{|c|c|c|c|c|c|}
\hline Disease category & Equally diagnostic & $\begin{array}{l}\text { TI-scout superior } \\
\text { to standard LGE }\end{array}$ & $\begin{array}{l}\text { Standard LGE superior } \\
\text { to TI-scout }\end{array}$ & Complementary & Total \\
\hline Unrepaired congenital heart diseases & $1(17 \%)$ & $3(50 \%)$ & 0 & $2(33 \%)$ & 6 \\
\hline Repaired congenital heart diseases & $1(4 \%)$ & $18(72 \%)$ & $3(12 \%)$ & $3(12 \%)$ & 25 \\
\hline Aortic valvular diseases & 0 & $3(100 \%)$ & 0 & 0 & 3 \\
\hline Hypertrophic cardiomyopathy & $1(10 \%)$ & $6(60 \%)$ & 0 & $3(30 \%)$ & 10 \\
\hline $\begin{array}{l}\text { Cardiomyopathy or myocarditis (exclud- } \\
\text { ing hypertrophic cardiomyopathy) }\end{array}$ & $5(16 \%)$ & $10(32 \%)$ & $10(32 \%)$ & $6(19 \%)$ & 31 \\
\hline Cardiac tumors & 0 & 0 & $1(100 \%)$ & 0 & 1 \\
\hline Systemic vascular diseases & $1(33 \%)$ & $1(33 \%)$ & 0 & $1(33 \%)$ & 3 \\
\hline Total & $9(11 \%)$ & $41(52 \%)$ & $14(18 \%)$ & $15(19 \%)$ & $79(100 \%$ \\
\hline
\end{tabular}

LGE late gadolinium enhancement, $T$ I inversion time 


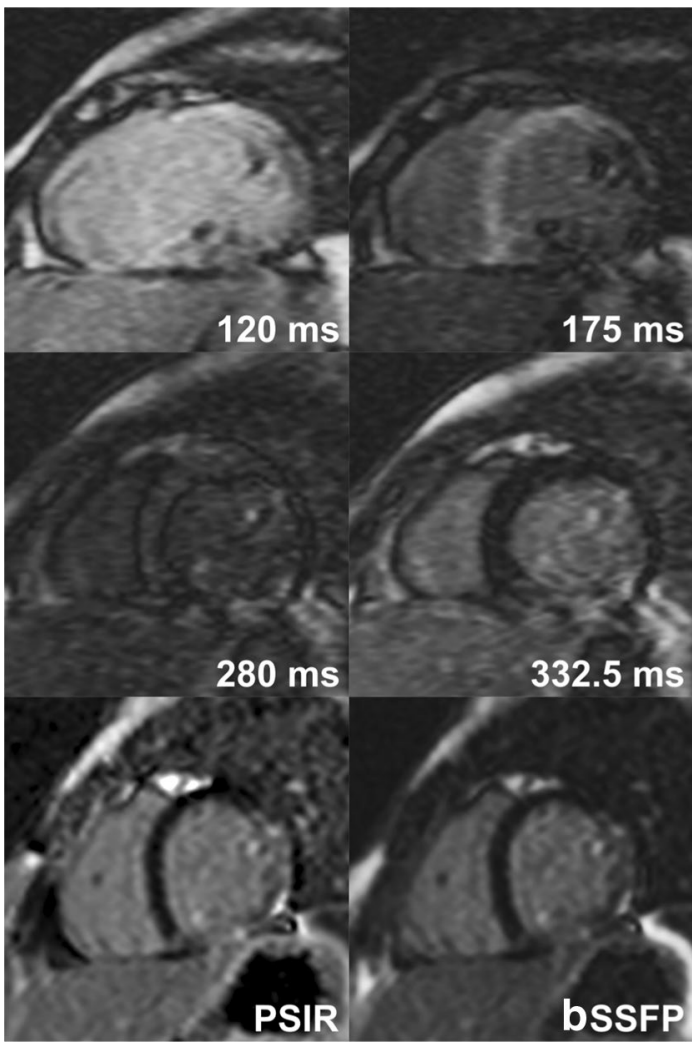

Fig. 2 Late gadolinium enhancement (LGE) in a 13-year old with Duchenne muscular dystrophy. A similar extent of LGE involving subepicaridal/mid-myocardial layer of the inferolateral and lateral wall and papillary muscles of the left ventricle is seen in both TI (inversion time)-scout and standard LGE images, playing complementary roles in the diagnosis of LGE. Phase sensitive inversion recovery (PSIR) and balanced steady-state free precession (bSSFP) standard LGE images were obtained at $\mathrm{Tl}$ of $330 \mathrm{~ms}$

TI-scout images in $9 \%$ of the cases with a negative diagnosis and $18 \%$ of those with a positive diagnosis. TI-scout images were more likely to be of incremental value in positive LGE group as opposed to negative LGE group $[\mathrm{OR}=6.49,95 \%$ CI $2.86-14.93, \mathrm{P}<0.001]$.

The diagnostic performance varied significantly according to the location of the LGE although TI-scout images were superior to standard LGE images in all locations (Table 6). TI-scout images were superior to standard LGE images in 11 of 12 (92\%) cases with LGE involving the papillary muscles (Fig. 3), in 7 of 12 (58\%) of the cases with endocardial-subendocardial LGE (Figs. 1 and 3), and in 4 of 7 (57\%) cases with transmural LGE. TI-scout images were particularly useful in making the diagnosis of the presence and extent of LGE in hypertrophic cardiomyopathy (Figs. 4 and 5), aortic valvular disease and repaired congenital heart disease. TI-scout was superior to standard LGE in $6 / 10(60 \%)$ and was complementary in $3 / 10(30 \%)$ of the positive cases with hypertrophic cardiomyopathy (Figs. 4 and 5, Table 5). TI-scout images were superior to standard LGE images in cases involving the endocardium-subendocardial myocardium (58\%) and papillary muscles (92\%) that included most cases with repaired congenital heart disease or aortic valvular diseases.

\section{Discussion}

LGE imaging is an established technique for detection of myocardial infarction and focal fibrosis [1]. However, there are limitations in detection of the lesions, particularly in areas of the myocardium adjacent to the blood pool and epicardial fat with standard LGE imaging. To overcome such limitations, the signal from the blood pool was completely or partially suppressed with or without T2 preparation between the inversion pulse and signal readout [4-7]. Although both complete and partial suppression techniques are helpful, neither technique is completely satisfactory. With complete suppression of the signal from the blood pool, localization of an enhancing lesion across the myocardium becomes difficult as the interface between the blood pool and myocardium

Table 6 Diagnostic performance of TI-scout versus standard LGE images in LGE positive cases by location

\begin{tabular}{|c|c|c|c|c|c|}
\hline LGE location & Equally diagnostic & $\begin{array}{l}\text { TI-scout superior } \\
\text { to standard LGE }\end{array}$ & $\begin{array}{l}\text { Standard LGE superior } \\
\text { to TI-scout }\end{array}$ & Complementary & Total \\
\hline Subepicardium & $4(14 \%)$ & $9(32 \%)$ & $8(29 \%)$ & $7(25 \%)$ & 28 \\
\hline Mid-myocardial & $1(6 \%)$ & $7(44 \%)$ & $3(19 \%)$ & $5(31 \%)$ & 16 \\
\hline $\begin{array}{l}\text { Endocardium and suben- } \\
\text { docardium }\end{array}$ & $1(8 \%)$ & $7(58 \%)$ & $2(17 \%)$ & $2(17 \%)$ & 12 \\
\hline Transmural & $1(14 \%)$ & $4(57 \%)$ & $1(14 \%)$ & $1(14 \%)$ & 7 \\
\hline Papillary muscle & $1(8 \%)$ & $11(92 \%)$ & 0 & 0 & 12 \\
\hline Mixed & $1(25 \%)$ & $3(75 \%)$ & 0 & 0 & 4 \\
\hline Total & $9(11 \%)$ & $41(52 \%)$ & $14(18 \%)$ & 15 (19\%) & 79 (100\%) \\
\hline
\end{tabular}

LGE late gadolinium enhancement, $T /$ inversion time 


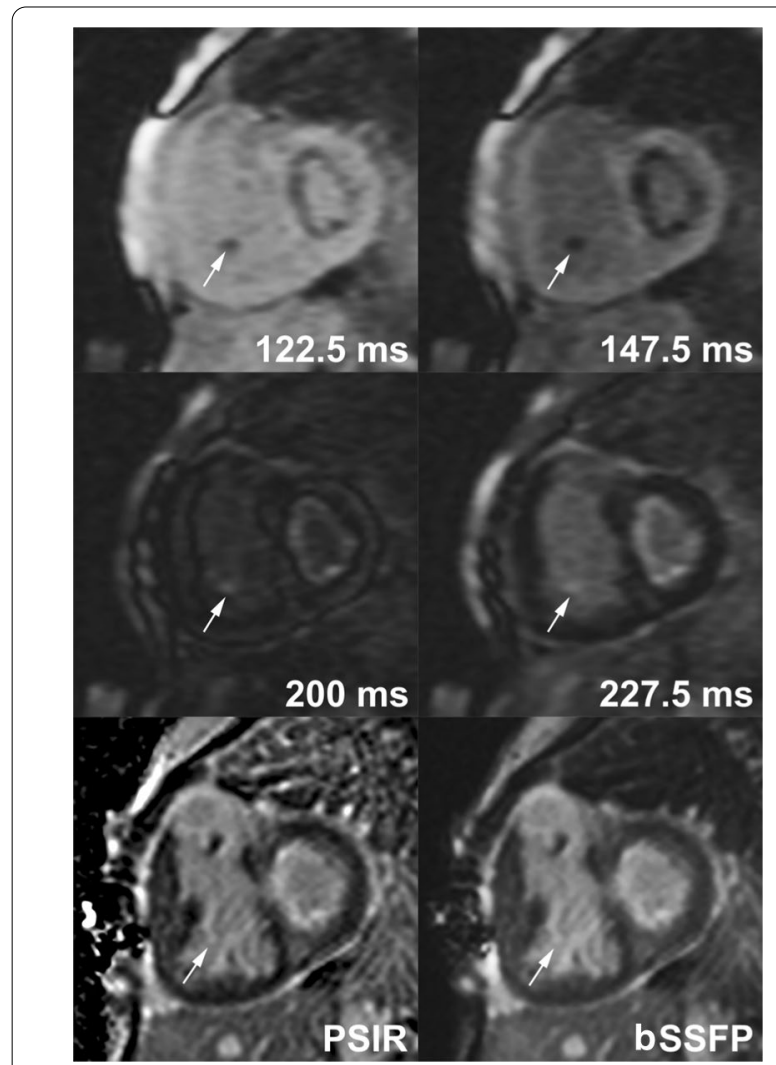

Fig. 3 Late gadolinium enhancement (LGE) in a 6-month old with critical aortic and mitral stenosis. The presence and extent of LGE involving endocardium and subendocardium is appreciated better in $\mathrm{Tl}$ (inversion time)-scout images than in phase sensitive inversion recovery (PSIR) and balanced steady state free precession (bSSFP) images. LGE of a papillary muscle (arrows) in the right ventricle is identifiable only in Tl-scout images. Phase sensitive inversion recovery (PSIR) and balanced steady-state free precession (bSSFP) standard LGE images were obtained at TI of $280 \mathrm{~ms}$ is lost. Partial suppression technique can also be limited in detection of myocardial lesions with signal intensity similar to that of the partially suppressed blood pool. In addition, the differentiation between the slow flow artifact along the endocardial surface and the true subendocardial or endocardial enhancement can be challenging.

In LGE imaging, T1-scout sequence is routinely acquired to identify the optimal myocardial nulling time. The acquired images show the changes in the signal intensity over time, that can be plotted visually or graphically. Usefulness of TI-scout imaging in the assessment of cardiomyopathies such as amyloid infiltration and hypertrophic cardiomyopathy has been reported [2, $3]$. In our experience in pediatric patients, we also found that these TI-scout images are helpful in determining the presence or absence of LGE when it is questionable or when LGE involves the endocardium, or subendocardial or subepicardial myocardium (Figs. 2 and 3). Based

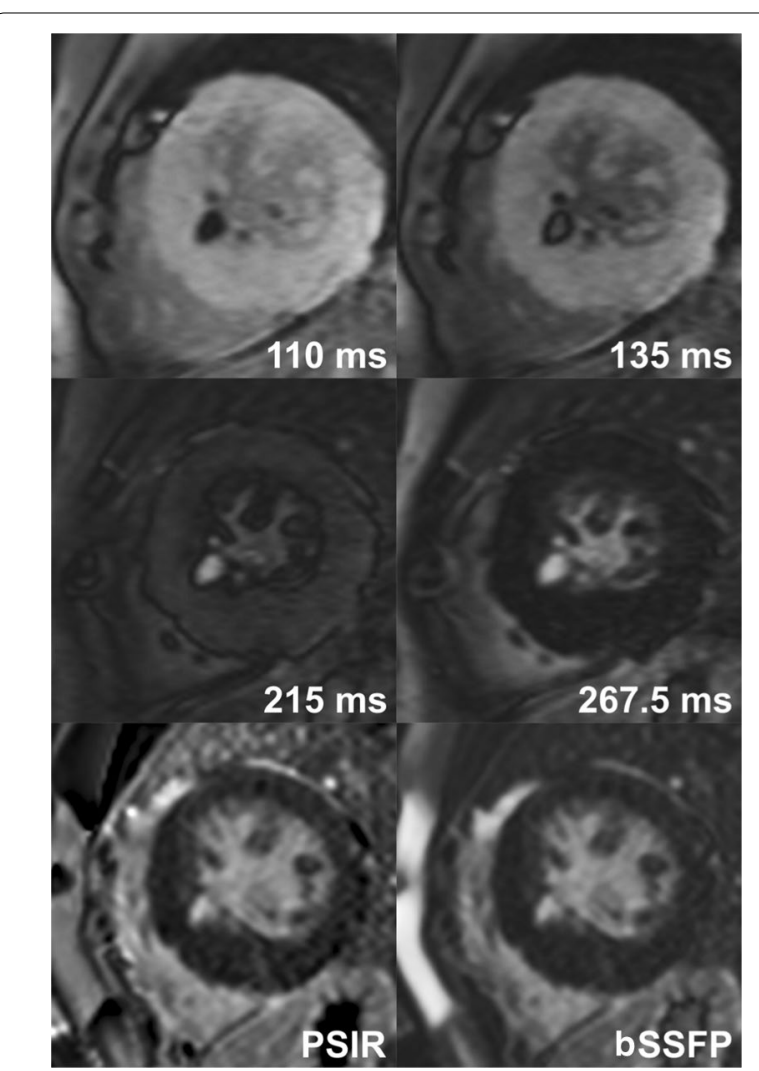

Fig. 4 Focal subendocardial fibrosis in a 14-year old with concentric hypertrophic cardiomyopathy. A focal subendocardial fibrosis is well appreciated in TI (inversion time)-scout images. In phase sensitive inversion recovery (PSIR) and balanced steady-state free precession (bSSFP) images, high signal intensity involving the same region may represent either true late gadolinium enhancement (LGE) or blood pool in a myocardial crypt. Phase sensitive inversion recovery (PSIR) and steady-state free precession (SSFP) standard LGE images were obtained at $\mathrm{Tl}$ of $310 \mathrm{~ms}$

on our initial experience, we started to acquire additional TI-scout images along the planes where the standard LGE images demonstrated inconclusive LGE. This retrospective analysis of our experience demonstrated: (1) TIscout and standard LGE images are equally diagnostic in excluding LGE, (2) TI-scout images are more often better than standard LGE in detecting LGE, (3) TI-scout and standard LGE images are often complementary in both negative and positive diagnosis of LGE, and (4) TI-scout images are particularly advantageous over standard LGE images in detecting the LGE involving the endocardium, subendocardial myocardium or papillary muscles, and LGE with low level enhancement such as so-called gray zone LGE or heterogeneous enhancement. The advantages of TI-scout in detection of LGE demonstrated in this study might not be well appreciated in pathologic conditions showing consistently strong LGE such as transmural myocardial infarction and, therefore, in adult 


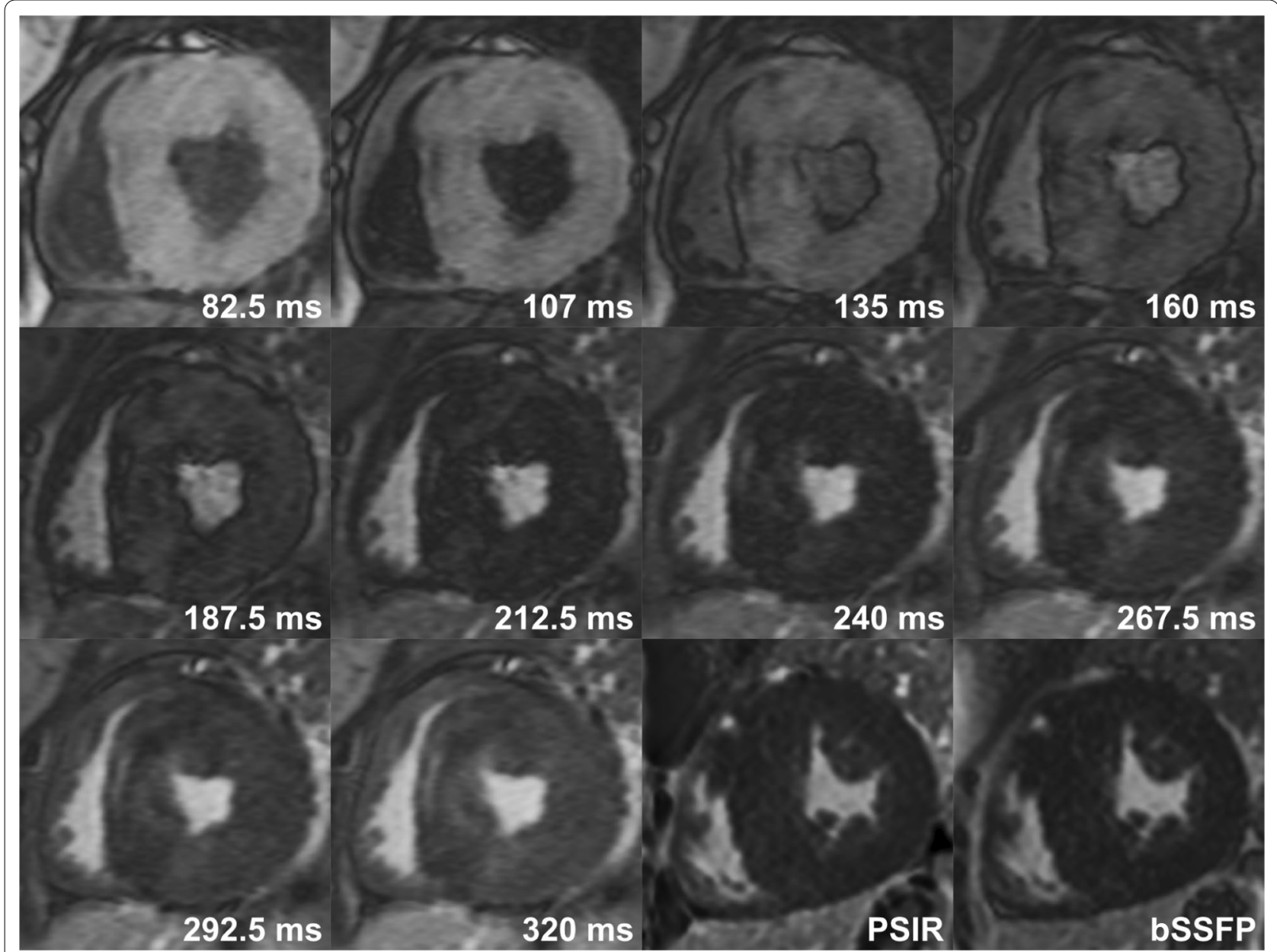

Fig. 5 Heterogeneous mild late gadolinium enhancement (LGE) in a 16-year old with with hypertrophic cardiomyopathy. Heterogeneity of the signal intensity of the hypertrophic myocardium can be confidently appreciated only in TI (inversion time)-scout images. PSIR and bSSFP standard LGE images were obtained at TI of $280 \mathrm{~ms}$

population. Furthermore, we emphasize that TI-scout imaging does not replace the standard LGE imaging but that TI-scout images augment LGE image interpretation.

Endocardial fibroelastosis is an important diagnosis in the newborns with left ventricular outflow tract obstruction and hypoplasia. Although it is associated with poor prognosis, it can be removed surgically to provide the left ventricle with an opportunity to grow for later biventricular repair [8]. In this regard, LGE imaging has been advocated as a valuable tool for accurate identification of endocardial fibroelastosis $[9,10]$. However, the sensitivity of LGE imaging for detection of endocardial fibroelastosis has not been established and even questioned [11]. In our study, TI-scout images were more diagnostic than standard LGE images in all 4 patients with endocardial fibroelastosis (Figs. 1 and 3). In standard LGE images, endocardial fibroelastosis did not stand out strongly against the bright blood pool unless LGE was distinctly strong. TI-scout images enabled the detection of LGE as they show the difference in gadolinium kinetics between normal myocardium, fibrotic myocardium and blood pool (Figs. 1 and 3). Similarly, TI-scout images were useful in clearly defining the transmural or subendocardial involvement of myocardial infarction.

LGE of the papillary muscle that was seen exclusively in patients with previous surgery in this study may represent fibrosis related to ischemia or chronic mechanical burden associated with atrioventricular valve regurgitation [12-15]. As the papillary muscles in the imaging plane may lack continuity with the remaining myocardium and are surrounded by the blood pool, LGE of the papillary muscles may escape detection with the standard LGE images alone because of the low contrast between the enhanced papillary muscles and blood pool. Therefore, complete or partial suppression of the blood pool signal was reported helpful in detection of LGE involving 
the papillary muscles $[4,6]$. In our study, T1-scout images played the major role in the detection of LGE involving the papillary muscles (Fig. 3). In making the diagnosis of LGE of the papillary muscles, it is important to be sure that the enhancing structure is not the chordae tendinae that normally enhance in LGE imaging by cross-referencing the cine images.

In patients with HCM, identification of LGE is important for risk stratification and is associated with increased risk for sudden cardiac death or appropriate discharge of implantable cardioverter defibrillator as well as heart failure [16-18]. The fibrotic myocardium in HCM typically is easily identifiable as areas with high signal intensities. However, it may also show low level LGE in the periphery of the strongly enhancing region $[19,20]$. Furthermore, the hypertrophic myocardium may show diffusely heterogeneous LGE without clearly identifiable margins. As the areas of low level LGE called gray zones may also serve as arrhythmogenic foci, proper assessment of the extent of the gray zones has been advocated $[19,20]$. In our study as well as in Amano et al. study, the areas of low level or heterogeneous signal intensities were much easier to perceive in TI-scout images than in standard LGE images. At least in one of the multiple TI-scout images, heterogeneity of the signal intensity is obvious and any uncertainty in standard LGE images can be eliminated with confidence (Fig. 5).

\section{Study limitations}

The study population was heterogeneous with the patients with various types of lesions, and the number of patients for each lesion was relatively small, which limited statistical analysis. The diagnostic performance of the TI-scout and standard LGE images were assessed qualitatively by visual comparison of the two sets of images but without any blinded assessment or pathological correlation. Lastly, utility of TI-scout in the assessment of the RV LGE was not able to be studied because of the small number of cases involving the RV. To assess the diagnostic impact of TI-scout images in assessing LGE in individual lesions, prospective quantitative studies are required using the pre-defined protocols including imaging parameters and planes in larger patient cohorts.

\section{Conclusions}

TI-scout images enhance the diagnostic performance of LGE imaging by playing the primary role or complementing the weakness of standard LGE images in the assessment of myocardial infarction or fibrosis. Therefore, active utilization of TI-scout imaging is advised. We propose routine acquisition of TI-scout images in three short-axis planes, vertical long axis planes of the RV and left ventricle, and 4-chamber plane. This approach would extend the exam by 3-4 min, and should be considered in addition to the standard LGE imaging so as to increase the confidence in standard LGE interpretation.

\section{Abbreviations}

bSSFP: Balanced steady-state free precession; CMR: Cardiovascular magnetic resonance; ECG: Electrocardiographic; FLASH: Fast low angle shot; LGE: Late gadolinium enhancement; MOLLI: Modified Look-Locker inversion recovery; PSIR: Phase-sensitive inversion recovery; RV: Right ventricle/right ventricular; TI: Inversion time.

\section{Acknowledgements}

The authors would like to acknowledge the support of Ontasian Imaging Lab (OIL).

\section{Authors' contributions}

All authors participated in imaging, data collection and analysis, manuscript preparation and approval of the content. All authors read and approved the final manuscript.

\section{Funding}

None.

Availability of data and materials

The datasets used and/or analysed during the current study are available from the corresponding author on reasonable request.

Ethics approval and consent to participate

Institutional Research Ethics (1000065145).

\section{Consent for publication}

Waived.

\section{Competing interests}

The authors declare that they have no competing interests.

\section{Author details}

${ }^{1}$ Department of Diagnostic Imaging, The Hospital for Sick Children, University of Toronto, 555 University Avenue, Toronto, ON M5G1X8, Canada. ${ }^{2}$ Department of Pediatrics, Division of Cardiology, Labatt Family Heart Centre, The Hospital for Sick Children, University of Toronto, Toronto, ON, Canada.

Received: 16 October 2020 Accepted: 31 January 2021

Published online: 18 March 2021

\section{References}

1. Kellman P, Arai AE. Cardiac imaging techniques for physicians: late enhancement. J Magn Reson Imaging. 2012;36:529-42.

2. Amano Y, Takayama M, Kumita S. Contrast-enhanced myocardial T1-weighted scout (Look-Locker) imaging for the detection of myocardial damages in hypertrophic cardiomyopathy. J Magn Reson Imaging. 2009:30:778-84.

3. Pandey T, Jambhekar K, Shaikh R, Lensing S, Viswamitra S. Utility of the inversion scout sequence (TI scout) in diagnosing myocardial amyloid infiltration. Int J Cardiovasc Imaging. 2013;29:103-12.

4. Kellman P, Xue H, Olivieri LJ, Cross RR, Grant EK, Fontana M, et al. Dark blood late enhancement imaging. J Cardiovasc Magn Reson. 2016;18:77.

5. Francis R, Kellman P, Kotecha T, Baggiano A, Norrington K, Martinez-Naharro A, et al. Prospective comparison of novel dark blood late gadolinium enhancement with conventional bright blood imaging for the detection of scar. J Cardiovasc Magn Reson. 2017;19:91.

6. Holtackers RJ, Van De Heyning CM, Nazir MS, Rashid I, Ntalas I, Rahman $\mathrm{H}$, et al. Clinical value of dark-blood late gadolinium enhancement cardiovascular magnetic resonance without additional magnetization preparation. J Cardiovasc Magn Reson. 2019;21:44. 
7. Fahmy AS, Neisius U, Tsao CW, Berg S, Goddu E, Pierce P, et al. Gray blood late gadolinium enhancement cardiovascular magnetic resonance for improved detection of myocardial scar. J Cardiovasc Magn Reson. 2018;20:22.

8. Emani SM, Bacha EA, McElhinney DB, Marx GR, Tworetzky W, Pigula FA, et al. Primary left ventricular rehabilitation is effective in maintaining twoventricle physiology in the borderline left heart. J Thorac Cardiovasc Surg. 2009;138:1276-82.

9. Stranzinger E, Ensing GJ, Hernandez RJ. MR findings of endocardial fibroelastosis in children. Pediatr Radiol. 2008;38:292-6.

10. Tworetzky W, del Nido PJ, Powell AJ, Marshall AC, Lock JE, Geva T. Usefulness of magnetic resonance imaging of left ventricular endocardial fibroelastosis in infants after fetal intervention for aortic valve stenosis. Am J Cardiol. 2005;96:1568-70.

11. Tuo G, Khambadkone S, Tann O, Kostolny M, Derrick G, Tsang V, et al. Obstructive left heart disease in neonates with a "borderline" left ventricle: diagnostic challenges to choosing the best outcome. Pediatr Cardiol. 2013;34:1567-76.

12. Yang Y, Connelly K, Graham JJ, Detsky J, Lee T, Walcarius R, et al. Papillary muscle involvement in myocardial infarction: initial results using multicontrast late-enhancement MRI. J Magn Reson Imaging. 2011;33:211-6.

13. Aldrovandi A, De Ridder SP, Strohm O, Cocker M, Sandonato R, Friedrich MG. Detection of papillary muscle infarction by late gadolinium enhancement: incremental value of short-inversion time vs. standard imaging. Eur Heart J Cardiovasc Imaging. 2013;14:495-9.

14. Enriquez A, Shirai Y, Huang J, Liang J, Briceño D, Hayashi T, et al. Papillary muscle ventricular arrhythmias in patients with arrhythmic mitral valve prolapse: electrophysiologic substrate and catheter ablation outcomes. J Cardiovasc Electrophysiol. 2019;30:827-35.
15. Fulton BL, Liang JJ, Enriquez A, Garcia FC, Supple GE, Riley MP, et al. Imaging characteristics of papillary muscle site of origin of ventricular arrhythmias in patients with mitral valve prolapse. J Cardiovasc Electrophysiol. 2018;29:146-53.

16. Maron BJ, Maron MS. LGE means better selection of HCM patients for primary prevention implantable defibrillators. JACC Cardiovasc Imaging. 2016;9:1403-6

17. Maron MS. The role of cardiovascular magnetic resonance in sudden death risk stratification in hypertrophic cardiomyopathy. Card Electrophysiol Clin. 2015;7:187-93.

18. Kramer CM, Neubauer S. Further refining risk in hypertrophic cardiomyopathy with late gadolinium enhancement by CMR. J Am Coll Cardiol. 2018;72:871-3.

19. Mesubi O, Ego-Osuala K, Jeudy J, Purtilo J, Synowski S, Abutaleb A, et al. Differences in quantitative assessment of myocardial scar and gray zone by LGE-CMR imaging using established gray zone protocols. Int J Cardiovasc Imaging. 2015;31:359-68.

20. Spiewak M, Małek LA, Chojnowska L, Miśko J, Petryka J, Kłopotowski M, et al. Late gadolinium enhancement gray zone in patients with hypertrophic cardiomyopathy. Comparison of different gray zone definitions. Int J Cardiovasc Imaging. 2010;26:693-9.

\section{Publisher's note}

Springer Nature remains neutral with regard to jurisdictional claims in published maps and institutional affiliations.
Ready to submit your research? Choose BMC and benefit from:

- fast, convenient online submission

- thorough peer review by experienced researchers in your field

- rapid publication on acceptance

- support for research data, including large and complex data types

- gold Open Access which fosters wider collaboration and increased citations

- maximum visibility for your research: over $100 \mathrm{M}$ website views per year

At BMC, research is always in progress.

Learn more biomedcentral.com/submissions 